\title{
On Association Cells in Random Heterogeneous Networks
}

\author{
Sarabjot Singh, François Baccelli, and Jeffrey G. Andrews
}

\begin{abstract}
Characterizing user to access point (AP) association strategies in heterogeneous cellular networks (HetNets) is critical for their performance analysis, as it directly influences the load across the network. In this letter, we introduce and analyze a class of association strategies, which we term stationary association, and the resulting association cells. For random HetNets, where APs are distributed according to a stationary point process, the area of the resulting association cells are shown to be the marks of the corresponding point process. Addressing the need of quantifying the load experienced by a typical user, a "Fellerparadox" like relationship is established between the area of the association cell containing origin and that of a typical association cell. For the specific case of Poisson point process and max power/SINR association, the mean association area of each tier is derived and shown to increase with channel gain variance and decrease in the path loss exponents of the corresponding tier.
\end{abstract}

\section{INTRODUCTION}

Densification of wireless cellular infrastructure through deployment of low power APs is a promising approach to meet increasing wireless traffic demands. This complementary infrastructure consists of various classes of APs differing in transmit powers, radio access technologies, backhaul capacities, and deployment scenarios. This increasing heterogeneity and density in wireless networks has provided an impetus to develop new models for their analysis and design. This paper is primarily aimed to analyze such random heterogeneous networks (HetNets), where the AP locations are modeled by a stationary point process.

Using Poisson point processes (PPP) for modeling the irregular AP locations has been shown to be a tractable and accurate approach for characterizing signal-to-interference ratio (SIR) distribution [1]-[3]. Although SIR is one of the key performance metrics for user performance, managing load or the number of users sharing the available resources per AP plays an important role in realizing the capacity gains in HetNets [4]. The load at an AP is dictated by the user to AP association strategy adopted in the network. For example, users associating to their nearest AP leads to association cells conforming to a Voronoi tessellation with AP locations as the cell centers and identical load distribution across the APs. However, in HetNets, it is desirable to incorporate the differing base station (BS) capabilities among the classes/tiers of BSs and the propagation environments in the association strategy.

This work has been supported by the Intel-Cisco Video Aware Wireless Networks (VAWN) Program and NSF grant CIF-1016649. The authors are with Dept. of Electrical and Computer Engineering at the University of Texas, Austin (email: sarabjot@utexas.edu, baccelli@math.utexas.edu, and jandrews@ece.utexas.edu).
Being able to characterize the resulting complex association cells is one of the goals of this paper.

In this paper, we introduce stationary association strategies, which lead to the formation of stationary association cells. Such strategies form a wide class and encompass all association patterns that are invariant by translation, including the earlier studied max SINR association [5]. Leveraging the theory of stationary partitions introduced in [6], we establish a "Feller-paradox" like relation between the association area of the AP containing the origin to that of a typical AP in a HetNet setting, wherein the former is an area-biased version of the latter. Such a relation has important practical implications in analyzing the load experienced by a typical user which is served, as we shall see, by an atypical AP. The developed theoretical framework also provides rigorous proofs for the arguments used in [7], [8] for load characterization. Further, using the PPP assumption and max-power association, it is shown that the association area of a typical AP of a tier increases with the channel gain variance and decrease in the path loss exponent for the corresponding tier.

\section{Stationary ASSOCIATION}

The locations of the base stations are $\left\{T_{n}\right\}$ and seen as the atoms of a stationary point process (PP) $\Phi$ defined on a measurable space $(\Omega, \mathcal{A}, \mathbb{P})$ and having intensity $\lambda$. The analysis in this paper is for $\mathbb{R}^{2}$ due to the practical implications, but it also extends to $\mathbb{R}^{d}$. Further $\Phi$ is assumed to be $\theta_{t}$ compatible, where $\theta_{t}$ is a measurable flow on $\Omega$, so that

$$
\Phi(\omega, B+x)=\Phi\left(\theta_{x} \omega, B\right), \omega \in \Omega, x \in \mathbb{R}^{2}, B \in \mathcal{B},
$$

where $\mathcal{B}$ denotes the Borel $\sigma$-field on $\mathbb{R}^{2}$. The operation $\theta_{x} \omega$ can also be thought of as $\Phi(\omega)$ shifted by $-x$. Let $\zeta(x) \in$ $\Phi \forall x \in \mathbb{R}^{2}$ denote the base station to which a user lying at $x$ associates. The mapping $\zeta: \Omega \times \mathbb{R}^{2} \rightarrow \mathbb{R}^{2}$ is referred to as an association strategy.

Definition 1. Stationary Association: An association strategy $\zeta(x)$ is stationary if the association is translation invariant, i.e.,

$$
\zeta(x)=\zeta(0) \circ \theta_{x} \forall x \in \mathbb{R}^{2},
$$

where $\circ$ denotes the composition operator.

Further a collection of fields $\left\{M_{n}(y)\right\} \in \mathbb{R}^{+} \cup \infty \forall y \in \mathbb{R}^{2}$ is assumed associated with the atoms $\left\{T_{n}\right\}$ of $\Phi$ such that

$$
\begin{aligned}
M_{0}(y) \circ \theta_{T_{n}} & =M_{n}\left(y+T_{n}\right) \text { and } \\
M_{n}(y) & =\infty \text { if } y=T_{n},
\end{aligned}
$$

and therefore for a given $y$, the associated field $M_{n}(y)$ forms a sequence of marks for $\Phi$. Define a mapping $\kappa(y) \triangleq$ 
$\arg \sup M_{n}(y)$, where the sup is assumed to be well defined. Thus, by definition 1 and $(2), \kappa(y)$ is a stationary association.

Definition 2. Association Cell: The association cell $\mathcal{C}\left(T_{n}\right)$ of an $\mathrm{AP}$ at $T_{n}$ is defined as

$$
\mathcal{C}\left(T_{n}\right)=\left\{y \in \mathbb{R}^{2}: \kappa(y)=T_{n}\right\}
$$

and $\left|\mathcal{C}\left(T_{n}\right)\right|$ is the corresponding association area.

Lemma 1. Under stationary association, the area of association cells is a sequence of marks.

Proof: It needs to be shown that $\left|\mathcal{C}\left(T_{n}\right)\right|=\left|\mathcal{C}\left(T_{0}\right) \circ \theta_{T_{n}}\right|$.

$$
\begin{aligned}
& \mathcal{C}\left(T_{0}\right) \circ \theta_{T_{n}}=\left\{y: M_{0}(y) \circ \theta_{T_{n}}>M_{m}(y) \circ \theta_{T_{n}} \forall m \neq 0\right\} \\
& \stackrel{(a)}{=}\left\{y: M_{n}\left(y+T_{n}\right)>M_{m}\left(y+T_{n}\right) \forall m^{\prime} \neq n\right\} \\
& =\mathcal{C}_{n}-T_{n},
\end{aligned}
$$

where (a) follows from (2). Since area is translation invariant the result follows.

Below are listed certain strategies that qualify as a stationary association under certain conditions.

I Max power association: User connects to the base station from which it receives the maximum power. Letting $P(n)$ denote the transmit power of AP at $T_{n}, H_{n}(y)$ denote the channel power gain and a power law path loss with path loss exponent $\alpha_{n}$, then the serving AP is

$$
\kappa(y)=\arg \sup S_{n}(y)=\arg \sup P(n) H_{n}(y)\left\|T_{n}-y\right\|^{-\alpha_{n}} .
$$

The field $S_{n}(y)$ satisfies 22 if $\left\{\alpha_{n}\right\}$ and $H_{n}(y)$ are a sequence of marks. Further, if $\arg \sup S_{n}(y)$ is well defined, then max power association is stationary 1

II Max SIR association: A user connects to the base station providing the highest SIR. The corresponding field is

$$
S_{n}(y)=\frac{P(n) H_{n}(y)\left\|T_{n}-y\right\|^{-\alpha_{n}}}{\sum_{m \neq n} P(m) H_{m}(y)\left\|T_{m}-y\right\|^{-\alpha_{m}}} .
$$

It can be seen that max SIR association is equivalent to max power association in I. Note that the association cells formed in this case are different than the SINR coverage cells defined in [9].

III Nearest base station association: This results in the classical case of Voronoi cells as association cells, which are stationary.

In this paper, the probability and expectation under the Palm probability are denoted by $\mathbb{P}^{o}$ and $\mathbb{E}^{o}[]$ respectively.

Proposition 1. For all measurable functions $f: \Omega \rightarrow \mathbb{R}^{+}$

$$
\mathbb{E}[f]=\lambda \mathbb{E}^{o}\left[\int_{\mathcal{C}\left(T_{0}\right)} f \circ \theta_{u} \mathrm{~d} u\right] .
$$

Proof: Stationary association $\kappa$ satisfying (1) can be seen as the stationary partition introduced in [6]. The proof follows using Theorem 4.1 of [6], which applies Mecke's formula to the function $h(\omega, x)=f(\omega) \mathbf{1}(x \in \Phi(\omega), \kappa(0)=x)$.

\footnotetext{
${ }^{1}$ If the sum $\sum_{n \geq 1} S_{n}(y)$ is finite almost surely (a.s.), then there exists no accumulation at $\sup S_{n}(y)$ a.s. and hence the $\arg \sup S_{n}(y)$ is well defined a.s.
}

\section{ASSOCIATION IN $K$-TIER NETWORKS}

In a $K$-tier HetNet, the APs are assumed to belong to $K$ distinct classes. Assuming independent deployment of APs of different tiers, we define i.i.d. marks mapping the AP index to tier index as $J\left(T_{n}\right) \in\{1 \ldots K\}$. The mapping distribution for a typical BS is

$$
p_{k} \triangleq \mathbb{P}^{o}\left(J\left(T_{0}\right)=k\right) .
$$

The location of the APs of $k^{\text {th }}$ tier is denoted by the point process $\Phi_{k}$, where

$$
\Phi_{k}=\sum_{T_{i} \in \Phi} \delta_{T_{i}} \mathbf{1}\left(J\left(T_{i}\right)=k\right) .
$$

The following proposition builds up on Prop. 1 to relate the probability of origin being contained in the association cell of tier $i$ to the association area of a typical cell of the corresponding tier.

Proposition 2. The probability that the origin is contained in the association cell of an atom of $\Phi_{i}$ is

$$
\mathcal{A}_{i} \triangleq \mathbb{P}(J(\kappa(0))=i)=\lambda p_{i} \mathbb{E}^{o, i}\left[\left|\mathcal{C}\left(T_{0}\right)\right|\right]
$$

Proof: Using Prop. 1 with $f=\mathbf{1}(J(\kappa(0))=i)$, we obtain

$$
\begin{gathered}
\mathbb{E}[\mathbf{1}(J(\kappa(0))=i)]=\lambda \mathbb{E}^{o}\left[\int_{\mathcal{C}\left(T_{0}\right)} \mathbf{1}\left(J(\kappa(0)) \circ \theta_{u}=i\right) \mathrm{d} u\right] \\
\mathbb{P}(J(\kappa(0))=i) \stackrel{(a)}{=} \lambda \mathbb{E}^{o}\left[\mathbf{1}(J(\kappa(0))=i)\left|\mathcal{C}\left(T_{0}\right)\right|\right] \\
\mathcal{A}_{i} \stackrel{(b)}{=} \lambda \mathbb{E}^{o, i}\left[\left|\mathcal{C}\left(T_{0}\right)\right|\right] \mathbb{P}^{o}(J(\kappa(0))=i),
\end{gathered}
$$

where (a) holds, as under palm $J(\kappa(0)) \circ \theta_{u}=J(\kappa(u))=$ $J(\kappa(0))$ for $u \in \mathcal{C}\left(T_{0}\right)$ and (b) follows from Bayes theorem.

For the case where users in the network form a homogeneous PPP, $\mathcal{A}_{i}$ denotes the probability of a typical user associating with the $i^{\text {th }}$ tier. The following proposition gives a conditional form of Prop. 1 in a $K$-tier setting.

Proposition 3. For all measurable functions $g: \Omega \rightarrow \mathbb{R}^{+}$

$$
\mathbb{E}[g \mid J(\kappa(0))=i]=\frac{\mathbb{E}^{o, i}\left[\int_{\mathcal{C}\left(T_{0}\right)} g \circ \theta_{u} \mathrm{~d} u\right]}{\mathbb{E}^{o, i}\left[\left|\mathcal{C}_{0}\right|\right]} .
$$

Proof: Using $f=g \mathbf{1}(J(\kappa(0))=i)$ in Prop. 1. the LHS is

$$
\mathbb{E}[g \mathbb{1}(J(\kappa(0))=i)]=\mathbb{E}[g \mid J(\kappa(0))=i] \mathcal{A}_{i},
$$

and the RHS is

$\lambda \mathbb{E}^{o}\left[\mathbb{1}(J(\kappa(0))=i) \int_{\mathcal{C}\left(T_{0}\right)} g \circ \theta_{u} \mathrm{~d} u\right]=\lambda p_{i} \mathbb{E}^{o, i}\left[\int_{\mathcal{C}\left(T_{0}\right)} g \circ \theta_{u} \mathrm{~d} u\right]$

Using Prop. 2 in the above, gives the result.

Using the above proposition, the density of association area of the AP of tier $i$ containing origin (assuming it exists) can be given in terms of that of the area of a typical association cell of the corresponding tier. 


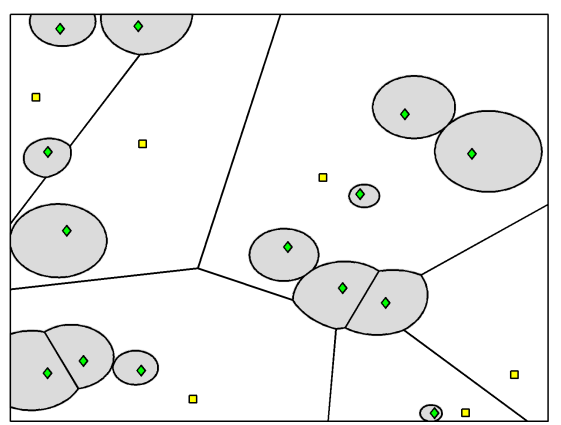

(a) No channel variance, $\sigma_{1}=\sigma_{2}=0$

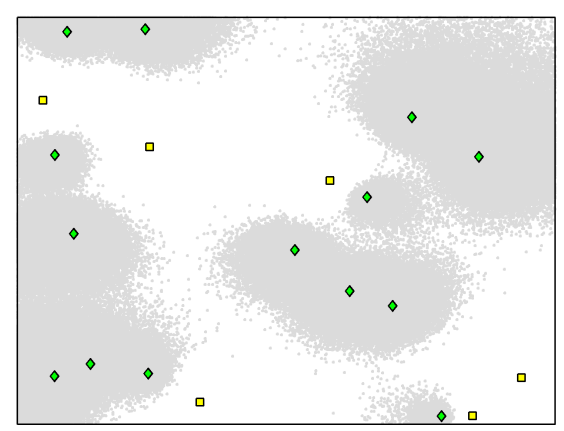

(b) Low channel variance, $\sigma_{1}=1, \sigma_{2}=1$

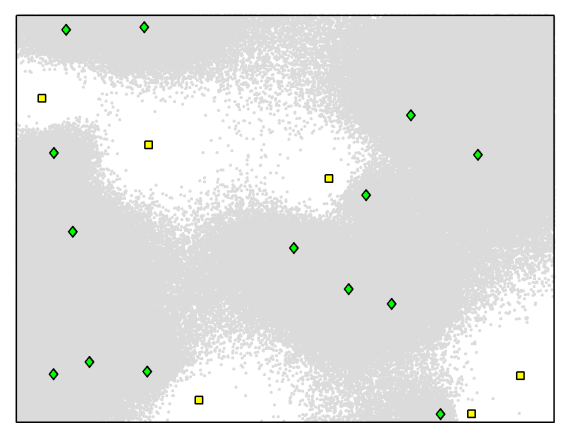

(c) High channel variance, $\sigma_{1}=1, \sigma_{2}=2$

Fig. 1: The shaded region is served by the APs of tier-2 (diamonds), while the rest of the area is served by tier-1 APs (squares).

Corollary 1. The density of the area of the association cell of tier $i$ containing origin is given by

$$
f_{|\mathcal{C}(\kappa(0))|}^{i}(c)=\frac{c f_{\left|\mathcal{C}\left(T_{0}\right)\right|}^{o, i}(c)}{\mathbb{E}^{o, i}\left[\left|\mathcal{C}\left(T_{0}\right)\right|\right]},
$$

where $f_{\left|\mathcal{C}\left(T_{0}\right)\right|}^{o, i}$ is the density of the area of a typical association cell of tier $i$.

Proof: Using $g=\mathbf{1}(v \leq|\mathcal{C}(\kappa(0))| \leq v+\mathrm{d} v)$ in Prop. 3 we get

$$
\begin{array}{r}
\mathbb{P}(c \leq|\mathcal{C}(\kappa(0))| \leq c+\mathrm{d} c \mid J(\kappa(0))=i) \\
=\frac{\mathbb{E}^{o, i}\left[\int_{\mathcal{C}\left(T_{0}\right)} \mathbf{1}\left(c \leq\left|\mathcal{C}\left(\kappa(0) \circ \theta_{u}\right)\right| \leq c+\mathrm{d} c\right) \mathrm{d} u\right]}{\mathbb{E}^{o, i}[|\mathcal{C}(\kappa(0))|]} \\
f_{|\mathcal{C}(\kappa(0))|}^{o, i}(c) \stackrel{(a)}{=} \frac{\mathbb{E}^{o, i}[\mathbf{1}(c \leq|\mathcal{C}(\kappa(0))| \leq c+\mathrm{d} c)|\mathcal{C}(\kappa(0))|]}{\mathbb{E}^{o, i}[|\mathcal{C}(\kappa(0))|]},
\end{array}
$$

where (a) follows from the fact that under the Palm distribution $\left|\mathcal{C}\left(\kappa(0) \circ \theta_{u}\right)\right|=|\mathcal{C}(\kappa(0))|$ for $u \in \mathcal{C}(\kappa(0))$. The final result is obtained using Bayes theorem and the fact that under the Palm distribution $\kappa(0)=T_{0}$.

As a consequence of the above corollary it can be stated that the area of the association cell containing a typical user is larger than that of a typical cell, and the following holds

$$
\left.\mathbb{E}\left[|\mathcal{C}(\kappa(0))|^{d} \mid J(\kappa(0))=i\right)\right]=\frac{\mathbb{E}^{o, i}\left[|\mathcal{C}(\kappa(0))|^{d+1}\right]}{\mathbb{E}^{o, i}[|\mathcal{C}(\kappa(0))|]} \forall d \in \mathbb{R}
$$

\section{Mean Association ARea in PPP HetNets}

In this section, the mean association area is derived for the case where the base station process $\Phi$ is assumed to be a PPP. The general max power/SINR association given in (3) is considered. It is further assumed that the APs of $k^{\text {th }}$ tier have the same constant power and path loss exponents, and have independent but identical channel gain distribution, i.e, $P(n)=\mathrm{P}_{k}, \alpha_{n}=a_{k}$, and $H_{n}(y) \stackrel{(d)}{=} H_{k} \forall T_{n} \in \Phi_{k}$. Due to the i.i.d. assumption on $J$ marks, by the thinning theorem [10], each tier process $\Phi_{k}$ is a PPP with density $\lambda_{k} \triangleq p_{k} \lambda$ for $k=1 \ldots K$. For illustration, Fig. 1 shows the association cells in a two tier setup with $\mathrm{P}_{1}=53 \mathrm{dBm}, \mathrm{P}_{2}=33 \mathrm{dBm}, a_{1}=$ $a_{2}=4$, and the channel gain is lognormal $H_{k} \sim \ln \mathcal{N}\left(0, \sigma_{k}\right)$. As seen from the plots, increasing the variance in the channel gain for the second tier increases the corresponding association areas (the shaded areas). This observation is made rigorous by the following analysis.

\section{A. Analysis}

Lemma 2. Under the max power association, the mean association area of a typical base station of the $i^{\text {th }}$ tier is

$$
2 \pi \int_{0}^{\infty} r \mathbb{E}_{H_{i}}\left[\exp \left(-\pi \sum_{k=1}^{K} \tilde{\lambda}_{k} r^{2 a_{i} / a_{k}} \mathrm{P}_{i}^{-2 / a_{k}} H_{i}^{-2 / a_{k}}\right)\right] \mathrm{d} r,
$$

where $\tilde{\lambda}_{k}=\lambda_{k} \mathrm{P}_{k}^{2 / a_{k}} \mathbb{E}\left[H_{k}^{2 / a_{k}}\right]$ and $\mathbb{E}\left[H_{k}^{2 / a_{k}}\right]<\infty$.

Proof: The mean association area of a typical cell of the $i^{\text {th }}$ tier is $\mathbb{E}^{o, i}\left[\left|\mathcal{C}\left(T_{0}\right)\right|\right]=\int_{\mathbb{R}^{2}} \mathbb{P}^{o, i}\left(u \in \mathcal{C}\left(T_{0}\right)\right) \mathrm{d} u$, which $\forall n \neq 0$

$$
\begin{aligned}
& =\mathbb{P}^{o, i}\left(\|u\|^{a_{i} / \alpha_{n}}\left(H(0, u) \mathrm{P}_{i}\right)^{-1 / \alpha_{n}}<\left\|T_{n}-u\right\|\left(H(n, u) \mathrm{P}_{n}\right)^{-1 / \alpha_{n}}\right) \\
& =\mathbb{P}^{o, i}\left(\bigcap_{k=1}^{K} \tilde{\Phi}_{k}\left(B^{o}\left(0,\|u\|^{a_{i} / a_{k}}\left(H(0, u) \mathrm{P}_{i}\right)^{-1 / a_{k}}\right)=0\right),\right.
\end{aligned}
$$

where $\tilde{\Phi}_{k}$ denotes the PPP formed by transforming the atoms of $\Phi_{k}: T_{n} \rightarrow\left(T_{n}-u\right)\left(H(n, u) \mathrm{P}_{k}\right)^{-1 / a_{k}}$. By the i.i.d. displacement theorem [10], $\tilde{\Phi}_{k}$ is a homogeneous PPP with $\tilde{\lambda}_{k}=\lambda_{k} \mathrm{P}_{k}^{2 / a_{k}} \mathbb{E}\left[H_{k}^{2 / a_{k}}\right]$, given $\mathbb{E}\left[H_{k}^{2 / a_{k}}\right]<\infty,[5],[11]$. Thus

$$
\begin{aligned}
& \mathbb{P}^{o, i}\left(u \in \mathcal{C}\left(T_{0}\right)\right)=\int_{0}^{\infty} \mathbb{P}^{o, i, h}\left(u \in \mathcal{C}\left(T_{0}\right)\right) f_{H_{i}}(h) \mathrm{d} h \\
& \stackrel{(a)}{=} \mathbb{E}_{H_{i}}\left[\prod_{k=1}^{K} \mathbb{P}^{o, i}\left(\tilde{\Phi}_{k}\left(B^{o}\left(0,\|u\|^{a_{i} / a_{k}}\left(H_{i} \mathrm{P}_{i}\right)^{-1 / a_{k}}\right)\right)=0\right)\right] \\
&=\mathbb{E}_{H_{i}}\left[\exp \left(-\pi \sum_{k=1}^{K} \tilde{\lambda}_{k}\|u\|^{2 a_{i} / a_{k}}\left(H_{i} \mathrm{P}_{i}\right)^{-2 / a_{k}}\right)\right]
\end{aligned}
$$

For the case with the path loss exponents of each tier being the same: $a_{k} \equiv a$, the mean association area simplifies to

$$
\mathbb{E}^{o, i}\left[\left|\mathcal{C}\left(T_{0}\right)\right|\right]=\frac{\mathrm{P}_{i}^{2 / a} \mathbb{E}\left[H_{i}^{2 / a}\right]}{\sum_{k=1}^{K} \lambda_{k} \mathrm{P}_{k}^{2 / a} \mathbb{E}\left[H_{k}^{2 / a}\right]}
$$


and thus depends on only the $\frac{2}{a}^{\text {th }}$ moment of the channel gain. Using Prop. 2 for association probability leads to the earlier derived result in [5], which used propagation invariance.

Remark 1. The framework developed above can be used to compute additive functionals over association cells. Using Campbell's theorem [10], the mean of an additive characteristic $g$ associated with a typical association cell of tier $i$ and defined on an independent PPP $\Phi_{u}$ of intensity $\lambda_{u}$ is

$$
\begin{array}{r}
\bar{S}=\mathbb{E}^{o, i}\left[\mathbb{E}_{\Phi_{u}}\left[\sum_{j} g\left(Y_{j}\right) \mathbf{1}\left(Y_{j} \in \mathcal{C}\left(T_{0}\right)\right)\right]\right] \\
=\int_{\mathbb{R}^{2}} g(y) \mathbb{P}^{o, i}\left(y \in \mathcal{C}\left(T_{0}\right)\right) \lambda_{u} \mathrm{~d} y .
\end{array}
$$

For example, if $\Phi_{u}$ represents the user point process and $g(x)=\|x\|^{-a}$ (a path loss function), then $\bar{S}$ represents the mean total power received at a typical AP of tier $i$ from all the users served by it and is given by (using (4))

$2 \pi \lambda_{u} \int_{r>0} r^{-a+1} \mathbb{E}_{H_{i}}\left[\exp \left(-\pi \sum_{k=1}^{K} \tilde{\lambda}_{k} r^{2 a_{i} / a_{k}}\left(H_{i} \mathrm{P}_{i}\right)^{-2 / a_{k}}\right)\right] \mathrm{d} r$

\section{B. Numerical Results}

We consider a two tier (macro and pico, say) setup along with max power association with respective transmit powers: $\mathrm{P}_{1}=53 \mathrm{dBm}$ and $\mathrm{P}_{2}=33 \mathrm{dBm}$. The variation in the mean association area with the variation in density of small cells (second tier) is shown in Fig. 2, where $\sigma_{1}=2$, $a_{1}=a_{2}=3.5, \lambda_{1}=1 \mathrm{BS} / \mathrm{sq}$. $\mathrm{Km}$, and the channel gains are assumed lognormal with $H_{k} \sim \ln \mathcal{N}\left(0, \sigma_{k}\right)$. It can be seen that with increasing variance in the channel propagation, the corresponding mean association area increases. This follows from 5 , and the fact that $\mathbb{E}\left[H_{k}^{2 / a}\right]=\exp \left(0.5(2 / a)^{2} \sigma_{k}^{2}\right)$. The effect of path loss exponent on the mean association area is shown in Fig. 3. For the plot, $\sigma_{1}=2, \sigma_{2}=4$, and $a_{1}=3$. As can be seen, with decreasing path loss exponent of small cells, the corresponding association area increases. Intuitively, the lower the path loss exponent, the lower the decay rate of the corresponding AP's transmission power and hence there will be a larger number of users associating with the same.

\section{CONClusion}

We introduce the notion of stationary association for random HetNets with the resulting association areas shown to be the marks of the corresponding point process. Analogous to a Voronoi tessellation, an inversion formula relating the association area of the cell containing origin to a typical association area for each tier is proved. It is shown that with max power association, the mean association area of small cells decreases with path loss exponent and increases with channel gain variance.

\section{REFERENCES}

[1] J. G. Andrews, F. Baccelli, and R. K. Ganti, "A tractable approach to coverage and rate in cellular networks," IEEE Trans. Commun., vol. 59, pp. 3122-3134, Nov. 2011.

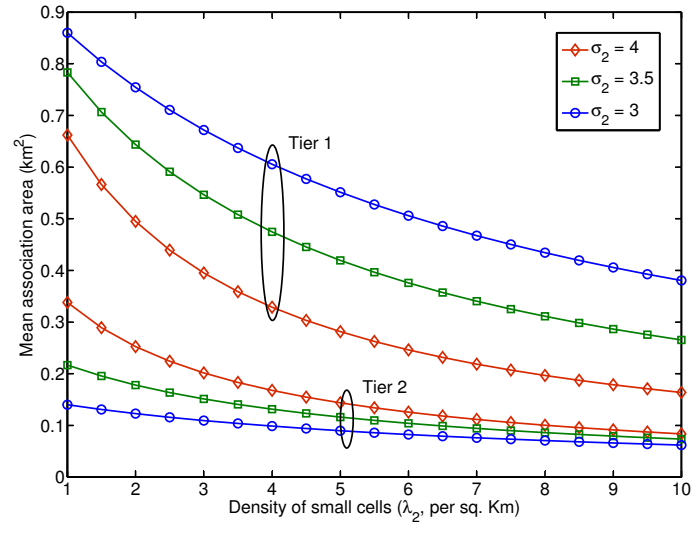

Fig. 2: Variation of mean association areas of two tiers with density for different channel gain variances of second tier

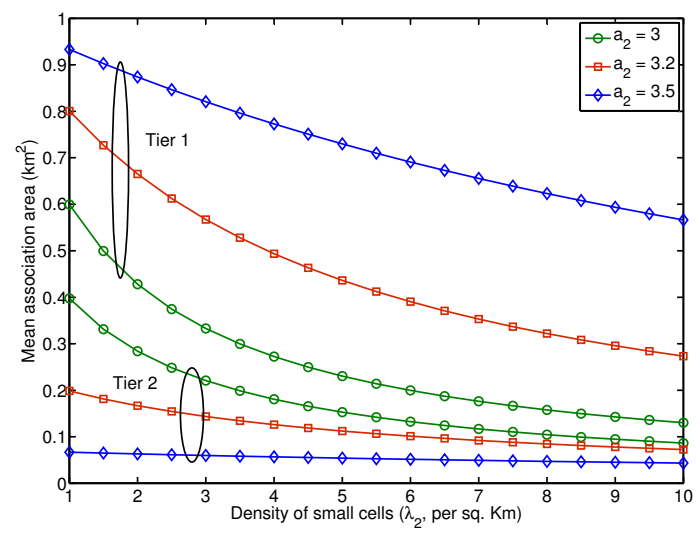

Fig. 3: Variation of mean association areas of two tiers with density for different path loss exponents of second tier

[2] B. Blaszczyszyn, M. K. Karray, and H.-P. Keeler, "Using Poisson processes to model lattice cellular networks," in Proc. IEEE INFOCOM, pp. 773-781, Apr. 2013.

[3] H. S. Dhillon, R. K. Ganti, F. Baccelli, and J. G. Andrews, "Modeling and analysis of $K$-tier downlink heterogeneous cellular networks," IEEE J. Sel. Areas Commun., vol. 30, pp. 550-560, Apr. 2012.

[4] J. G. Andrews et al., "An overview of load balancing in HetNets: Old myths and open problems," IEEE Wireless Commun. Mag., submitted. Available at: http://arxiv.org/abs/1307.7779.

[5] P. Madhusudhanan, J. Restrepo, Y. Liu, and T. Brown, "Downlink coverage analysis in a heterogeneous cellular network," in IEEE GLOBECOM, pp. 4170-4175, Dec. 2012.

[6] G. Last, "Stationary partitions and Palm probabilities," Advances in Applied Probability, vol. 38, pp. 602-620, Sept. 2006.

[7] S. Singh, H. S. Dhillon, and J. G. Andrews, "Offloading in heterogeneous networks: Modeling, analysis, and design insights," IEEE Trans. Wireless Commun., vol. 12, pp. 2484-2497, May 2013.

[8] S. Singh and J. G. Andrews, "Joint resource partitioning and offloading in heterogeneous cellular networks," IEEE Trans. Wireless Commun., submitted. Available at: http://arxiv.org/abs/1303.7039.

[9] F. Baccelli and B. Błaszczyszyn, "On a coverage process ranging from the boolean model to the Poisson-Voronoi tessellation with applications to wireless communications," Advances in Applied Probability, vol. 33, pp. pp. 293-323, June 2001.

[10] F. Baccelli and B. Blaszczyszyn, Stochastic Geometry and Wireless Networks, Volume I - Theory. NOW: Foundations and Trends in Networking, 2009.

[11] B. Błaszczyszyn and H. P. Keeler, "Equivalence and comparison of heterogeneous cellular networks," in International WDN Workshop on Cooperative and Heterogeneous Cellular Networks, Sept. 2013. 